\title{
Some relations between complex structures on compact nilmanifolds and flag manifolds
}

\author{
Takumi YAMADA \\ (Received March 31, 2020) \\ (Revised May 26, 2021)
}

\begin{abstract}
In this paper, we first consider relations between signatures of pseudoKähler metrics on a flag manifold and complex structures on a nilpotent Lie algebra corresponding to the flag manifold. On the nilpotent Lie algebra, we also consider complex structures which do not correspond to invariant complex structures on the flag manifold.
\end{abstract}

\section{Introduction}

In this paper, we first consider relations between signatures of pseudoKähler metrics on a flag manifold and complex structures on a nilpotent Lie algebra corresponding to the flag manifold. For the flag manifold $M=$ $S U(3) / T^{2}$, there exist invariant pseudo-Kähler metrics of type $(6,0),(0,6)$, $(4,2)$, and $(2,4)([9])$. On the other hand, for a 6-dimensional nilpotent Lie algebra $\mathbb{R}_{\mathbb{R}}\left(\mathfrak{h}(1)^{\mathbb{C}}\right)$, where $\mathbb{R}_{\mathbb{R}}\left(\mathfrak{h}(1)^{\mathbb{C}}\right)$ is the scalar restriction of 3-dimensional complex Heisenberg algebra $\mathfrak{h}(1)^{\mathbb{C}}$, there exist distinct 4 connected components of the modular space $\mathscr{C}\left(\mathbb{R}\left(\mathfrak{h}(1)^{\mathbb{C}}\right)\right.$ ) (see [8] for details). Let $T_{o}^{\mathbb{C}} M$ be the complexification of tangent space of the point $o=e T^{2}$, and $T^{1,0} M$ the complex eigendistribution of the complex structure $J$ with an eigenvalue $\sqrt{-1}$. Then, $T_{o}^{1,0} M$ can be identified with a complex nilpotent Lie algebra $\mathfrak{h}(1)^{\mathbb{C}}=$ $\left(\mathbb{R}\left(\mathfrak{h}(1)^{\mathbb{C}}\right), J\right)$.

In previous papers, we considered signatures of pseudo-Kähler metrics on a flag manifold and complex structures on a nilpotent Lie algebra, separately. Let $\mathfrak{g}$ be a real Lie algebra, and $\mathfrak{g}=\mathfrak{a}+\mathfrak{b}$ a direct sum decomposition such that $\mathfrak{a}$ and $\mathfrak{b}$ are Lie subalgebras of $\mathfrak{g}$. Then, we can construct an integrable complex structure $\tilde{J}$ on $\mathbb{R}_{(}\left(\mathfrak{g}^{\mathbb{C}}\right)$ from the decomposition. Then, we studied relations between the decomposition and $\operatorname{dim} H_{\bar{\partial}_{\tilde{J}}}^{s, t}\left(\mathbb{R}_{\mathbb{R}}\left(\mathfrak{g}^{\mathbb{C}}\right)\right)$ for investigating the complex structure $\tilde{J}$ (see e.g. [10, Theorems 3.2, 3.3]). On the other hand, in

The author is supported by JSPS KAKENHI Grant Number JP20K03586.

2010 Mathematics Subject Classification. Primary 22E25; Secondary 53C30, 17 B22.

Key words and phrases. nilmanifold, root system, complex structure. 
the paper [9], we considered the signatures of pseudo-Kähler metrics on the full flag manifolds.

More precisely, we now consider the case of root system $A_{2}$. Let $\left\{\alpha_{1}, \alpha_{2}\right\}$ be a basis of $A_{2}$ with natural manner ([4]). By using results of previous papers ([11, Section 4], [9]), we have the following relations among Weyl chambers of the root system $A_{2}$, left-invariant complex structures on $\mathbb{R}_{\mathbb{R}}\left(\mathfrak{h}(1)^{\mathbb{C}}\right)$, and signatures of pseudo-Kähler metrics on $M=S U(3) / T^{2}$.

\begin{tabular}{|c|c|c|c|c|}
\hline Weyl chamber & \multicolumn{3}{|c|}{ complex structure of nilpotent Lie group } & signature \\
\hline$C_{0}=\left\{\alpha_{1}>0, \alpha_{2}>0\right\}$ & $N_{0}=\{$ & $\left.\begin{array}{cc}z_{1} & z_{3} \\
1 & z_{2} \\
0 & 1\end{array}\right)$ & $z_{i} \in \mathbb{C}$ & $(6,0)$ \\
\hline$C_{1}=\left\{-\alpha_{1}>0, \alpha_{1}+\alpha_{2}>0\right\}$ & $N_{1}=\{$ & $\left.\begin{array}{cc}\bar{z}_{1} & z_{3} \\
1 & z_{2} \\
0 & 1\end{array}\right) \mid$ & $z_{i} \in \mathbb{C}$ & $(4,2)$ \\
\hline$C_{2}=\left\{-\alpha_{2}>0, \alpha_{1}+\alpha_{2}>0\right\}$ & $N_{2}=\left\{\left(\begin{array}{l}1 \\
0 \\
0\end{array}\right.\right.$ & $\begin{array}{c}w_{3}+w_{1} \bar{u} \\
\bar{w}_{2} \\
1\end{array}$ & $\left.\left.\bar{v}_{2}\right) \mid w_{i} \in \mathbb{C}\right\}$ & $(4,2)$ \\
\hline$C_{3}=\left\{\alpha_{1}>0,-\alpha_{1}-\alpha_{2}>0\right\}$ & $N_{3}=\{$ & $\left.\begin{array}{cc}z_{1} & \bar{z}_{3} \\
1 & \bar{z}_{2} \\
0 & 1\end{array}\right)$ & $z_{i} \in \mathbb{C}$ & $(2,4)$ \\
\hline$C_{4}=\left\{\alpha_{2}>0,-\alpha_{1}-\alpha_{2}>0\right\}$ & $N_{4}=\left\{\begin{array}{l}1 \\
0 \\
0\end{array}\right.$ & $\begin{array}{c}\bar{w}_{3}+\bar{w}_{1} n \\
w_{2} \\
1\end{array}$ & $\left.\left.v_{2}\right) \mid w_{i} \in \mathbb{C}\right\}$ & $(2,4)$ \\
\hline$C_{5}=\left\{-\alpha_{1}>0,-\alpha_{2}>0\right\}$ & $N_{5}=\{$ & $\left.\begin{array}{cc}\bar{z}_{1} & \bar{z}_{3} \\
1 & \bar{z}_{2} \\
0 & 1\end{array}\right)$ & $\left.z_{i} \in \mathbb{C}\right\}$ & $(0,6)$ \\
\hline
\end{tabular}

There exist holomorphic isomorphisms $f_{12}: N_{1} \rightarrow N_{2}, f_{34}: N_{3} \rightarrow N_{4}$, and antiholomorphic isomorphisms $f_{05}: N_{0} \rightarrow N_{5}, f_{13}: N_{1} \rightarrow N_{3}, f_{24}: N_{2} \rightarrow N_{4}$. Thus, $N_{0}, N_{1} \cong N_{2}, N_{3} \cong N_{4}$ and $N_{5}$ are not holomorphically isomorphic each other. On the other hand, we have a symmetry of the signatures of invariant pseudo-Kähler metrics. In this paper we generalize those relations.

On a real nilpotent Lie algebra given by the scalar restriction of a complex nilpotent Lie algebra $T_{o}^{1,0} M$ of a flag manifold $M$, we also consider complex structures $\tilde{J}$ which do not correspond to invariant complex structures on the flag manifold $M$. However, we use Weyl chambers for constructing complex structures on the real nilpotent Lie algebra (See Sections 5 and 6). For 
example, the nilpotent Lie group with a left-invariant complex structure defined by

$$
\left\{\left(\begin{array}{cccc}
1 & \bar{x}_{1} & x_{2} & z \\
0 & 1 & 0 & y_{1} \\
0 & 0 & 1 & y_{2} \\
0 & 0 & 0 & 1
\end{array}\right) \mid x_{1}, x_{2}, y_{1}, y_{2}, z \in \mathbb{C}\right\}
$$

does not correspond to an invariant complex structure on the flag manifold $S U(4) / T^{2} \times S U(2)$.

Let $\mathbb{R}_{\mathbb{R}}\left(\mathrm{n}^{\mathbb{C}}\right)$ be a nilpotent Lie algebra constructed from a root system $A_{l}$.

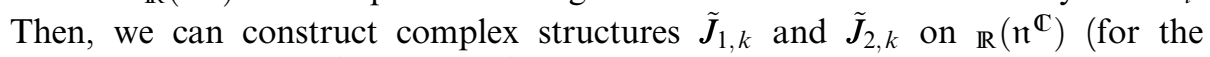
details of $\mathbb{R}_{\mathbb{R}}\left(\mathfrak{n}^{\mathbb{C}}\right), \quad \tilde{J}_{1, k}$ and $\tilde{J}_{2, k}$, see Sections 4 and 5$)$. We denote $\operatorname{dim} H_{\hat{\partial}_{J}}^{s, t}\left({ }_{\mathbb{R}}\left(\mathfrak{n}^{\mathbb{C}}\right)\right)$ by $h^{s, t}\left(\mathfrak{n}_{J}\right)$. Then, we show the following result:

THEOREM 8. Let $\tilde{J}_{1, k}, \tilde{J}_{2, k}$ be complex structures on $\mathbb{R}_{\mathbb{R}}\left(\mathfrak{n}^{\mathbb{C}}\right)$ corresponding to decompositions of roots induced by subsets $\Pi_{1, k}, \Pi_{2, k}$ of a basis of a root system $A_{l}$, respectively. Then,

$$
\sum_{s+t=r} h^{s, t}\left(\mathfrak{n}_{\tilde{J}_{1, k-1}}\right)=\sum_{s+t=r} h^{s, t}\left(\mathfrak{n}_{\tilde{J}_{2, k+1}}\right)
$$

for each $k$ and $r$.

\section{Preliminaries}

In this section, we recall an integrability condition of an almost leftinvariant complex structure on a Lie group, and relations between Dolbeault cohomology groups of a nilmanifold with a complex structure and cohomology groups of a nilpotent Lie algebra.

On the integrability condition, we have

THEOREM 1 (Andrada-Salamon [2]). Let $\mathfrak{g}$ be a Lie algebra with a complex structure $J$ which satisfies $J[X, Y]=[J X, Y]$ for all $X, Y \in \mathfrak{g}$. Suppose there exists a splitting $\mathfrak{g}=\mathfrak{u}_{1}+\mathfrak{u}_{2}$ with complex subalgebras $\mathfrak{u}_{1}, \mathfrak{u}_{2}$ of $\mathfrak{g}$. Then the linear endomorphism $\tilde{J}$ defined by

$$
\left.\tilde{J}\right|_{\mathfrak{u}_{1}}=-J,\left.\quad \tilde{J}\right|_{\mathfrak{u}_{2}}=J
$$

is a complex structure on $\mathfrak{g}$.

Let $N$ be a simply connected real nilpotent Lie group. It is well known that there exists a lattice in $N$ if and only if there exists a rational Lie subalgebra $\mathfrak{n}_{\mathbb{Q}}$ such that $\mathfrak{n} \cong \mathfrak{n}_{\mathbb{Q}} \otimes \mathbb{R}$ (cf. [6]). Then, a complex structure $J$ 
on $\mathrm{n}$ is said to be rational if $J\left(\mathfrak{n}_{\mathbb{Q}}\right) \subset \mathfrak{n}_{\mathbb{Q}}([5])$. Then, we have the following results.

THEOREM 2 (Console-Fino [5]). Let $N$ be a simply connected nilpotent Lie group, and $\Gamma$ a lattice in $N$. If $J$ is a $\Gamma$-rational complex structure on $\mathrm{n}$, then

$$
H_{\bar{\partial}}^{s, t}(\Gamma \backslash N) \cong H_{\bar{\partial}}^{s, t}\left(\mathrm{n}^{\mathbb{C}}\right)
$$

for each $s, t$.

THeOrem 3 (Console-Fino [5]). For any small deformation of a $\Gamma$-rational complex structure, the isomorphism

$$
H_{\bar{\partial}}^{s, t}(\Gamma \backslash N) \cong H_{\bar{\partial}}^{s, t}\left(\mathrm{n}^{\mathbb{C}}\right)
$$

holds for each $s$, $t$.

THEOREM 4 (Sakane [7]). Let $N$ be a simply connected complex nilpotent Lie group, and $\Gamma$ a lattice in $N$. Then,

$$
H_{\bar{\partial}}^{s, t}(\Gamma \backslash N) \cong H_{\bar{\partial}}^{0, t}\left(\mathrm{n}^{-}\right) \otimes \bigwedge^{s}\left(\mathrm{n}^{+}\right)^{*} \cong H^{t}\left(\mathrm{n}^{-}\right) \otimes \bigwedge^{s}\left(\mathrm{n}^{+}\right)^{*}
$$

for each $s$, $t$.

Thus, results on $H_{\bar{\partial}}^{s, t}\left(\mathfrak{n}^{\mathbb{C}}\right)$ of a nilpotent Lie algebra with good complex structures yield results on $H_{\bar{\partial}}^{s, t}(\Gamma \backslash N)$ of a compact nilmanifold with invariant complex structures.

\section{Flag manifolds and Nilpotent Lie algebras}

In this section, we first consider relations between signatures of pseudoKähler metrics on a flag manifold and complex structures on a nilpotent Lie algebra corresponding to the flag manifold. For details of notions of $T$-root systems, see [1], [3].

Let $G$ be a compact semi-simple Lie group, $\mathfrak{g}$ the Lie algebra of $G$, and $\mathfrak{h}$ a maximal abelian subalgebra. We identify an element of the root system $R$ of $\mathfrak{g}^{\mathbb{C}}$ relative to the Cartan subalgebra $\mathfrak{h}^{\mathbb{C}}$ with an element of $\mathfrak{h}_{0}=\sqrt{-1} \mathfrak{h}$ by the duality defined by the Killing form of $\mathfrak{g}^{\mathbb{C}}$. We consider the following root system decomposition relative to $\mathfrak{h}^{\mathbb{C}}$ :

$$
\mathfrak{g}^{\mathbb{C}}=\mathfrak{h}^{\mathbb{C}}+\sum_{\alpha \in R} \mathfrak{g}_{\alpha}^{\mathbb{C}} .
$$

Definition 1. (1) A subset $Q \subset R$ is said to be closed if for each $\alpha, \beta \in Q$ with $\alpha+\beta \in R$, it holds $\alpha+\beta \in Q$.

(2) A subset $Q \subset R$ is said to be asymmetric if $Q \cap(-Q)=\varnothing$. 
Let $\Pi=\left\{\alpha_{1}, \ldots, \alpha_{l}\right\}$ be a basis of the root system $R$. We denote by $R^{+}$the set of all positive roots relative to $\Pi$. Let $\Pi_{0}$ be a subset of $\Pi$ and $\Pi-\Pi_{0}=\left\{\alpha_{i_{1}}, \ldots, \alpha_{i_{r}}\right\}$, where $1 \leq i_{1}<\cdots<i_{r} \leq l$. We put $\mathrm{t}=\left\{H \in \mathfrak{h}_{0} \mid\right.$ $\left.\left(H, \Pi_{0}\right)=0\right\}$. Then,

$$
\mathrm{n}^{\mathbb{C}}=\sum_{\alpha \in R^{+}-\left[\Pi_{0}\right]} \mathfrak{g}_{\alpha}^{\mathbb{C}}
$$

is a nilpotent Lie algebra, where $\left[\Pi_{0}\right]=R \cap\left\{\Pi_{0}\right\}_{\mathbb{Z}}$. We put $R_{\mathrm{m}}=R-\left[\Pi_{0}\right]$ and $R_{\mathfrak{m}}^{+}=R_{\mathfrak{m}} \cap R^{+}$. Take a Weyl basis $E_{\alpha} \in \mathfrak{g}_{\alpha}^{\mathbb{C}}(\alpha \in R)$. Then, the structure constants $N_{\alpha, \beta}$ satisfy $N_{\alpha, \beta}=N_{-\alpha,-\beta} \in \mathbb{R}$, where $\left[E_{\alpha}, E_{\beta}\right]=N_{\alpha, \beta} E_{\alpha+\beta}$ if $\alpha, \beta, \alpha+\beta \in R$. Let $\mathfrak{g}_{\alpha}=\mathbb{R} E_{\alpha}$, and $\mathfrak{n}=\sum_{\alpha \in R_{\mathrm{m}}^{+}} \mathfrak{g}_{\alpha}$.

We consider the restriction map

$$
\kappa:\left.\mathfrak{h}_{0} \rightarrow \mathfrak{t}^{*} \quad \alpha \mapsto \alpha\right|_{\mathfrak{t}}
$$

and set $R_{T}=\kappa(R)$. The elements of $R_{T}$ are called T-roots. The collection of hyperplanes $\{\kappa(\alpha)=0\}$ corresponding to $T$-roots decomposes the space $\mathrm{t}$ into a finite number of cones, which are called $T$-chambers. We denote by $B(C)$ a basis of $\mathrm{t}^{*}$ corresponding to a $T$-chamber $C$. We denote by $C_{0}$ a chamber $\left\{\kappa\left(\alpha_{i_{1}}\right)>0, \ldots, \kappa\left(\alpha_{i_{r}}\right)>0\right\}$. We also denote by $R_{T}^{+}(C)$ the set of the positive $T$-roots corresponding to a $T$-chamber $C$.

Let $G^{\mathbb{C}}$ be a simply connected complex semi-simple Lie group and $U$ the parabolic subgroup of $G^{\mathbb{C}}$. Then the homogeneous complex manifold $G^{\mathbb{C}} / U$ is compact and simply connected, and $G$ acts transitively on $G^{\mathbb{C}} / U$. Note that $K=G \cap U$ is a connected closed subgroup of $G$, and $G^{\mathbb{C}} / U=G / K=M$ as $C^{\infty}$-manifolds. Let $\mathrm{m}$ be the orthogonal complement of the Lie algebra $\mathfrak{f}$ of $K$ with respect to the negative of the Killing form of $\mathfrak{g}$.

There exists a one-to-one correspondence between $T$-roots $\xi$ and irreducible submodules $\mathrm{m}_{\xi}$ of the $A d_{G}(K)$-module $\mathfrak{m}^{\mathbb{C}}$ given by

$$
R_{T} \ni \xi \mapsto \mathrm{m}_{\xi}=\sum_{\kappa(\alpha)=\xi} \mathfrak{g}_{\alpha}^{\mathbb{C}} .
$$

Thus, we have a decomposition of the $\operatorname{Ad}_{G}(K)$-module $\mathrm{m}^{\mathbb{C}}$ :

$$
\mathrm{m}^{\mathbb{C}}=\sum_{\xi \in R_{T}} \mathrm{~m}_{\xi} .
$$

We will identify $\mathrm{m}^{\mathbb{C}}$ with the complexification $T_{o}^{\mathbb{C}} M$ of tangent space $T_{o} M$ at the point $o=e K$.

THEOREM 5 (cf. [1]). There exist natural one-to-one correspondences between 
(1) $T$-bases $\Pi_{T}=\left\{\xi_{1}, \ldots, \xi_{r}\right\}$;

(2) $T$-chambers $C=\left\{\xi_{1}>0, \ldots, \xi_{r}>0\right\}$;

(3) systems $\Pi=\left\{\left(\xi_{1}\right)_{-}, \ldots,\left(\xi_{r}\right)_{-}\right\} \cup \Pi_{0}$ of simple roots of $R$ which contain fixed system $\Pi_{0}$, where $\left(\xi_{i}\right)_{-}$is the lowest weight of irreducible $A d_{G}(K)$-module $\mathrm{m}_{\xi_{i}}$ for each $i$;

(4) decomposition $R_{\mathrm{m}}=R_{+} \cup R_{-}$into disjoint union asymmetric closed subsets $R_{+}$and $R_{-}=-R_{+}$;

(5) invariant complex structures on the flag manifold $G / K$ (up to a sign).

In particular, for a decomposition $R_{\mathfrak{m}}=R_{+} \cup R_{-}$, we define a decomposition of the complexified tangent space $T_{o}^{\mathbb{C}} M=\mathrm{m}^{\mathbb{C}}=\mathrm{m}^{1,0}+\mathrm{m}^{0,1}$, where $\mathfrak{m}^{1,0}=\sum_{\alpha \in R_{+}} \mathfrak{g}_{\alpha}^{\mathbb{C}}, \quad \mathfrak{m}^{0,1}=\sum_{\alpha \in R_{-}} \mathfrak{g}_{\alpha}^{\mathbb{C}}$. Since the subspaces $\mathfrak{m}^{1,0}, \mathfrak{m}^{0,1}$ are $A d_{G}(K)$-invariant, they can be extended to two complex invariant distributions $T^{1,0} M$ and $T^{0,1} M$. We define an invariant complex structure $J$ on $M$ such that $T^{1,0} M$ and $T^{0,1} M$ are eigendistributions of $J$ with eigenvalues $+\sqrt{-1}$ and $-\sqrt{-1}$, respectively. Since $\mathfrak{f}^{\mathbb{C}}+\mathrm{m}^{1,0}$ is a subalgebra of $\mathfrak{g}^{\mathbb{C}}$, we have $J$ is integrable, where $\mathfrak{f}^{\mathbb{C}}=\mathfrak{h}^{\mathbb{C}}+\sum_{\alpha \in\left[\Pi_{0}\right]} \mathfrak{g}_{\alpha}^{\mathbb{C}}$.

Conversely, any invariant complex structure $J$ of $M=G / K$ defines a decomposition

$$
\mathrm{m}^{\mathbb{C}}=\mathrm{m}^{1,0}+\mathrm{m}^{0,1}, \quad \tau \mathrm{m}^{1,0}=\mathrm{m}^{0,1},
$$

where $\tau$ is the complex conjugation of $\mathfrak{g}^{\mathbb{C}}$ with respect to $\mathfrak{g}$. We denote by $J_{C}$ the complex structure on $\mathrm{m}^{\mathbb{C}}$ corresponding to a $T$-chamber $C$. Note that $J_{C}=+\sqrt{-1}$ id on $\mathrm{m}^{1,0}$, and $J_{C}=-\sqrt{-1}$ id on $\mathrm{m}^{0,1}$.

Let $C$ be a $T$-chamber. Put

$$
R_{\mathfrak{m}}^{A}=\left\{\alpha \in R_{\mathfrak{m}}^{+} \mid \kappa(\alpha) \in R_{T}^{-}(C)\right\}, \quad R_{\mathfrak{m}}^{B}=\left\{\alpha \in R_{\mathfrak{m}}^{+} \mid \kappa(\alpha) \in R_{T}^{+}(C)\right\} .
$$

Then, $R_{\mathfrak{m}}^{A}$ and $R_{\mathfrak{m}}^{B}$ are closed because $\kappa(\alpha)+\kappa(\beta)=\kappa(\alpha+\beta)$ for $\alpha, \beta \in R_{\mathfrak{m}}^{+}$. Thus we have a direct sum decomposition $\mathfrak{n}^{\mathbb{C}}=\mathfrak{a}^{\mathbb{C}}+\mathfrak{b}^{\mathbb{C}}$, where $\mathfrak{a}^{\mathbb{C}}$ and $\mathfrak{b}^{\mathbb{C}}$ are complex Lie subalgebras defined by

$$
\mathfrak{a}^{\mathbb{C}}=\sum_{\alpha \in R_{\mathrm{m}}^{A}} \mathfrak{g}_{\alpha}^{\mathbb{C}}, \quad \mathfrak{b}^{\mathbb{C}}=\sum_{\alpha \in R_{\mathrm{m}}^{B}} \mathfrak{g}_{\alpha}^{\mathbb{C}} .
$$

Thus, we can consider a complex structure $\tilde{J}$ on $\mathbb{R}_{\mathbb{R}}\left(\mathfrak{n}^{\mathbb{C}}\right)$ defined by

$$
\tilde{J}= \begin{cases}-J & \text { on } \mathbb{R}\left(\mathfrak{a}^{\mathbb{C}}\right) \\ J & \text { on } \mathbb{R}\left(\mathfrak{b}^{\mathbb{C}}\right) .\end{cases}
$$

Because $\mathfrak{a}^{\mathbb{C}}=\mathfrak{n}^{\mathbb{C}} \cap \mathfrak{m}^{0,1}$, and $\mathfrak{b}^{\mathbb{C}}=\mathfrak{n}^{\mathbb{C}} \cap \mathfrak{m}^{1,0}$, where $\mathfrak{m}^{\mathbb{C}}=\mathfrak{m}^{1,0}+\mathfrak{m}^{0,1}$ is the decomposition corresponding to $J_{C}$, we have the following. 
THEOREM 6. Let $C$ be a T-chamber. Then,

$$
\tilde{J}=\left.J_{C}\right|_{\mathfrak{n}^{\mathbb{C}}} .
$$

Proof. This follows from the fact that, if $Z$ is an eigenvector of $J$ with the eigenvalue $+\sqrt{-1}$, then $Z$ is an eigenvector of $-J$ with the eigenvalue $-\sqrt{-1}$.

Therefore, we can use classifications of invariant complex structures of a flag manifold for classifications of left-invariant complex structures of a nilpotent Lie group corresponding to the flag manifold.

Proposition 1. There exists a Weyl chamber $C^{\prime}$ such that

$$
\mathfrak{a}^{\mathbb{C}}=\sum_{\alpha \in R_{\mathrm{m}}^{A}} \mathfrak{g}_{\alpha}^{\mathbb{C}}=\sum_{\alpha \in R_{\mathrm{m}}^{+} \cap R^{-}\left(C^{\prime}\right)} \mathfrak{g}_{\alpha}^{\mathbb{C}}, \quad \mathfrak{b}^{\mathbb{C}}=\sum_{\alpha \in R_{\mathrm{m}}^{B}} \mathfrak{g}_{\alpha}^{\mathbb{C}}=\sum_{\alpha \in R_{\mathrm{m}}^{+} \cap R^{+}\left(C^{\prime}\right)} \mathfrak{g}_{\alpha}^{\mathbb{C}} .
$$

Proof. Put

$$
R_{+}=R_{\mathrm{m}}^{B} \cup\left[\Pi_{0}\right]^{+} \cup\left(-R_{\mathrm{m}}^{A}\right), \quad R_{-}=-R_{+},
$$

where $\left[\Pi_{0}\right]^{+}=\left[\Pi_{0}\right] \cap R^{+}$. Then, we see $R=R_{+} \cup R_{-}, R_{+} \cap R_{-}=\varnothing$. Let $\beta \in R_{\mathfrak{m}}^{B},-\alpha \in-R_{\mathfrak{m}}^{A}$, and suppose that $\beta+(-\alpha) \in R$. Since $\kappa(\beta-\alpha) \in R_{T}^{+}(C)$ and $R=R_{+} \cup R_{-}$, we have $\beta-\alpha \in R_{\mathrm{m}}^{B}$ or $\beta-\alpha \in-R_{\mathrm{m}}^{A}$. The other cases are trivial. Thus, $R_{+}$is closed. Since $R_{+}$is closed, there exists a Weyl chamber $C^{\prime}$ which satisfies $R_{+}=R^{+}\left(C^{\prime}\right)$ and $R_{-}=R^{-}\left(C^{\prime}\right)$ ([4]; Chapter 6, Corollary 1 of Proposition 20). Then,

$$
R_{\mathfrak{m}}^{A}=R_{\mathfrak{m}}^{+} \cap R_{-}=R_{\mathfrak{m}}^{+} \cap R^{-}\left(C^{\prime}\right), \quad R_{\mathfrak{m}}^{B}=R_{\mathfrak{m}}^{+} \cap R_{+}=R_{\mathfrak{m}}^{+} \cap R^{+}\left(C^{\prime}\right) .
$$

For integers $j_{1}, \ldots, j_{r}$ with $\left(j_{1}, \ldots, j_{r}\right) \neq(0, \ldots, 0)$, we put

$$
R\left(j_{1}, \ldots, j_{r}\right)=\left\{\sum_{j=1}^{l} m_{j} \alpha_{j} \in R^{+} \mid m_{i_{1}}=j_{1}, \ldots, m_{i_{r}}=j_{r}\right\} .
$$

Note that

$$
R_{\mathrm{m}}^{+}=R^{+}-\left[\Pi_{0}\right]=\bigcup_{j_{1}, \ldots, j_{r}} R\left(j_{1}, \ldots, j_{r}\right)
$$

We denote $m\left(j_{1}, \ldots, j_{r}\right)=\sharp R\left(j_{1}, \ldots, j_{r}\right)$, where $\sharp R\left(j_{1}, \ldots, j_{r}\right)$ means the number of elements of $R\left(j_{1}, \ldots, j_{r}\right)$.

We denote by $\omega_{\alpha}(\alpha \in R)$ the complex linear forms on $\mathfrak{g}^{\mathbb{C}}$ dual to the basis vectors $E_{\alpha}$ :

$$
\omega_{\alpha}\left(E_{\beta}\right)=\delta_{\alpha \beta}, \quad \omega_{\alpha}\left(\mathfrak{h}^{\mathbb{C}}\right)=\{0\}
$$


There exists a natural isomorphism $\mathrm{t}^{*} \rightarrow H^{2}(G / K, \mathbb{R})$ given by the formula

$$
\mathrm{t}^{*} \ni \lambda \rightarrow \eta(\lambda)=-\frac{1}{2 \pi \sqrt{-1}} d \lambda=-\frac{\sqrt{-1}}{2 \pi} \sum_{\alpha \in R_{\mathrm{m}}^{+}}(\lambda, \alpha) \omega_{-\alpha} \wedge \bar{\omega}_{-\alpha}
$$

where we consider $\lambda$ as a complex linear form on $\mathfrak{g}$ by extending.

Let $\lambda \in \mathrm{t}^{*}$, and $C$ the $T$-chamber corresponding to $\lambda$. Then,

$$
\begin{aligned}
\eta(\lambda) & =-\frac{\sqrt{-1}}{2 \pi} \sum_{\alpha \in R_{\mathrm{m}}^{+}}(\lambda, \alpha) \omega_{-\alpha} \wedge \bar{\omega}_{-\alpha} \\
& =-\frac{\sqrt{-1}}{2 \pi}\left(\sum_{\substack{\alpha \in R_{\mathrm{m}}^{+} \\
\kappa(\alpha) \in R_{T}^{+}(C)}}(\lambda, \alpha) \omega_{-\alpha} \wedge \bar{\omega}_{-\alpha}+\sum_{\substack{\alpha \in R_{\mathrm{m}}^{+} \\
\kappa(\alpha) \in R_{T}^{\mathrm{m}}(C)}}(\lambda, \alpha) \omega_{-\alpha} \wedge \bar{\omega}_{-\alpha}\right) .
\end{aligned}
$$

Note that $(\lambda, \cdot)$ is constant on $R\left(j_{1}, \ldots, j_{r}\right)$. Thus, the signature of $\eta(\lambda)$ can be written as

$$
\begin{aligned}
2(\sharp\{\alpha & \left.\left.\in R_{\mathfrak{m}}^{+} \mid \kappa(\alpha) \in R_{T}^{+}(C)\right\}, \sharp\left\{\alpha \in R_{\mathfrak{m}}^{+} \mid \kappa(\alpha) \in R_{T}^{-}(C)\right\}\right) \\
& =2\left(\sum_{\xi \in R_{T}^{+}\left(C_{0}\right) \cap R_{T}^{+}(C)} \operatorname{dim}_{\mathbb{C}} \mathfrak{m}_{\xi}, \sum_{\xi \in R_{T}^{+}\left(C_{0}\right) \cap R_{T}^{-}(C)} \operatorname{dim}_{\mathbb{C}} \mathrm{m}_{\xi}\right) .
\end{aligned}
$$

We have the following:

Proposition 2. Let $C$ be a T-chamber, and $\lambda \in C$. Then, the signature of $\eta(\lambda)$ can be written as

$$
2\left(\sum_{\xi \in R_{T}^{+}\left(C_{0}\right) \cap R_{T}^{+}(C)} \operatorname{dim}_{\mathbb{C}} \mathrm{m}_{\xi}, \sum_{\xi \in R_{T}^{+}\left(C_{0}\right) \cap R_{T}^{-}(C)} \operatorname{dim}_{\mathbb{C}} \mathrm{m}_{\xi}\right)=2\left(\operatorname{dim}_{\mathbb{C}} \mathfrak{b}^{\mathbb{C}}, \operatorname{dim}_{\mathbb{C}} \mathfrak{a}^{\mathbb{C}}\right) .
$$

Corollary 1. Let $C_{1}, C_{2}$ be $T$-chambers, and $\lambda_{1} \in C_{1}, \lambda_{2} \in C_{2}$. Let $\mathfrak{n}^{\mathbb{C}}=\mathfrak{a}_{1}^{\mathbb{C}}+\mathfrak{b}_{1}^{\mathbb{C}}, \mathfrak{n}^{\mathbb{C}}=\mathfrak{a}_{2}^{\mathbb{C}}+\mathfrak{b}_{2}^{\mathbb{C}}$ be decompositions corresponding to T-chambers $C_{1}$ and $C_{2}$, respectively. If signatures of $\eta\left(\lambda_{1}\right)$ and $\eta\left(\lambda_{2}\right)$ are different, then there exist no linear mapping $f_{*}: \mathfrak{n}^{\mathbb{C}} \rightarrow \mathfrak{n}^{\mathbb{C}}$ such that $f_{*}\left(\mathfrak{a}_{1}^{\mathbb{C}}\right)=\mathfrak{a}_{2}^{\mathbb{C}}$ and $f_{*}\left(\mathfrak{b}_{1}^{\mathbb{C}}\right)=$ $\mathfrak{b}_{2}^{\mathbb{C}}$.

We consider the case where $R$ is of $A_{l}$-type. Note that $\kappa(R)$ is also a root system $A_{r}$. Let $\varepsilon$ be the automorphism of $R=A_{l}$ that transforms $\alpha_{i}$ to $\alpha_{l+1-i}$. 
Assume that $\varepsilon\left(\Pi_{0}\right)=\Pi_{0} . \quad$ Then,

$$
\varepsilon\left(R\left(j_{1}, \ldots, j_{r}\right)\right)=R\left(j_{r}, \ldots, j_{1}\right),
$$

which implies $m\left(j_{1}, \ldots, j_{r}\right)=m\left(j_{r}, \ldots, j_{1}\right)$. Thus, we have the following:

Proposition 3. Suppose that $R$ is of $A_{l}$-type. Assume that $\varepsilon\left(\Pi_{0}\right)=\Pi_{0}$. Let $C_{1}, C_{2}$ be $T$-chambers, and $\lambda_{1} \in C_{1}, \lambda_{2} \in C_{2}$. If $\varepsilon\left(C_{1}\right)=C_{2}$, then the signatures of $\eta\left(\lambda_{1}\right)$ and $\eta\left(\lambda_{2}\right)$ are equal.

Proof. By assumption, we have $\sharp R_{T}^{+}\left(C_{0}\right) \cap R_{T}^{ \pm}\left(C_{1}\right)=\sharp R_{T}^{+}\left(C_{0}\right) \cap R_{T}^{ \pm}\left(C_{2}\right)$. Since $\operatorname{dim}_{\mathbb{C}} \mathfrak{m}_{\xi}=\operatorname{dim}_{\mathbb{C}} \mathfrak{m}_{\varepsilon(\xi)}$, we have our proposition.

\section{The case of root systems $A_{l}, D_{l}$, and $E_{6}$}

In this section, we consider complex structures on a nilpotent Lie algebra given by the scalar restriction of $T_{0}^{1,0} M$ of a flag manifold $M$. We mainly consider cases of root systems $A_{l}, D_{l}$, and $E_{6}$. From now on, we take a Chevalley basis $E_{\alpha} \in \mathfrak{g}_{\alpha}^{\mathbb{C}}(\alpha \in R)$. Then, structure constants $N_{\alpha, \beta}$ satisfy $N_{\alpha, \beta}=$ $-N_{-\alpha,-\beta}$, and $N_{\alpha, \beta} \in \mathbb{Z}$, where $\left[E_{\alpha}, E_{\beta}\right]=N_{\alpha, \beta} E_{\alpha+\beta}$ if $\alpha, \beta, \alpha+\beta \in R$.

Let $\Pi=\left\{\alpha_{1}, \ldots, \alpha_{l}\right\}$ be a basis of a root system $R$. Let $\Pi_{0}$ be a subset of $\Pi$. Put $\mathfrak{n}_{0}=\sum_{\alpha \in R^{+}} \mathfrak{g}_{\alpha}$. Let $C$ be the Weyl chamber corresponding to $\left\{\alpha_{1}, \ldots, \alpha_{l}\right\}$. Let $C^{\prime}$ be a Weyl chamber. Then, we have a decomposition $R=R^{+}\left(C^{\prime}\right) \cup R^{-}\left(C^{\prime}\right)$. Put $\quad R_{A}=R^{+}(C) \cap R^{-}\left(C^{\prime}\right), \quad R_{B}=R^{+}(C) \cap R^{+}\left(C^{\prime}\right)$. Let

$$
\mathfrak{a}=\sum_{\alpha \in R_{A}} \mathfrak{g}_{\alpha}, \quad \mathfrak{b}=\sum_{\alpha \in R_{B}} \mathfrak{g}_{\alpha}
$$

Then, we have

Proposition 4. The sets $\mathfrak{a}$ and $\mathfrak{b}$ are subalgebras of $\mathfrak{n}$ which satisfy $\mathfrak{n}=\mathfrak{a}+\mathfrak{b}$ and $\mathfrak{a} \cap \mathfrak{b}=\{\mathbf{0}\}$.

Proof. We have that $R_{A}$ and $R_{B}$ are closed because $R^{+}(C), R^{+}\left(C^{\prime}\right)$ and $R^{-}\left(C^{\prime}\right)$ are closed.

Thus, we can consider a complex structure $\tilde{J}$ on $\mathbb{R}_{\mathbb{R}}\left(\mathrm{n}^{\mathbb{C}}\right)$ corresponding to the decomposition $\mathfrak{n}=\mathfrak{a}+\mathfrak{b}$.

Put $\mathfrak{a}\left(\mathfrak{g}^{\mathbb{C}}\right)=\left\{\sigma \in \operatorname{Aut}\left(\mathfrak{g}^{\mathbb{C}}\right) \mid \sigma\left(\mathfrak{h}^{\mathbb{C}}\right)=\mathfrak{h}^{\mathbb{C}}\right\}$, and $A(R)=\left\{\hat{\sigma} \in \operatorname{Aut}\left(\mathfrak{h}^{*}\right) \mid \hat{\sigma}(R)=\right.$ $R\}$. Let $\mathfrak{i}\left(\mathfrak{g}^{\mathbb{C}}\right) \subset \mathfrak{a}\left(\mathfrak{g}^{\mathbb{C}}\right)$ be the set of the inner automorphisms, and $W(R)$ the Weyl group of $R$. Then, the following isomorphism are well-known:

$$
\mathfrak{a}\left(\mathfrak{g}^{\mathbb{C}}\right) / \mathfrak{i}\left(\mathfrak{g}^{\mathbb{C}}\right) \cong A(R) / W(R) \cong\{\varphi \in A(R) \mid \varphi(\Pi)=\Pi\} .
$$


For root systems $A_{l}, D_{l}$, and $E_{6}$, there exists a non-indentity map $\varepsilon \in$ $\{\varphi \in A(R) \mid \varphi(\Pi)=\Pi\}$. Let $\sigma$ be an element of $\mathfrak{a}\left(\mathfrak{g}^{\mathbb{C}}\right)$ which induces $\varepsilon$ by the above isomorphisms. Then, $\sigma$ induces an isomorphism $f_{*}=\left.\sigma\right|_{\mathfrak{n}_{0}^{\mathbb{C}}}: \mathfrak{n}_{0}^{\mathbb{C}} \rightarrow$ $\mathfrak{n}_{0}^{\mathbb{C}}$ because $\varepsilon(\Pi)=\Pi$ implies $\varepsilon\left(R^{+}\right)=R^{+}$.

Lemma 1. Let $C_{1}$ and $C_{2}$ be Weyl chambers. If $\varepsilon\left(R^{+}\left(C_{1}\right)\right)=R^{+}\left(C_{2}\right)$, then $\varepsilon\left(R^{-}\left(C_{1}\right)\right)=R^{-}\left(C_{2}\right)$.

Proof. Since $\varepsilon(R)=R$, we have $\varepsilon\left(R^{+}\left(C_{1}\right)\right) \cup \varepsilon\left(R^{-}\left(C_{1}\right)\right)=R^{+}\left(C_{2}\right) \cup$ $R^{-}\left(C_{2}\right)$.

Let $C_{1}$ and $C_{2}$ be Weyl chambers. Put

$$
\mathfrak{a}_{i}^{\mathbb{C}}=\sum_{\alpha \in R_{\mathfrak{m}}^{+} \cap R^{-}\left(C_{i}\right)} \mathfrak{g}_{\alpha}^{\mathbb{C}}, \quad \mathfrak{b}_{i}^{\mathbb{C}}=\sum_{\alpha \in R_{\mathfrak{m}}^{+} \cap R^{+}\left(C_{i}\right)} \mathfrak{g}_{\alpha}^{\mathbb{C}}
$$

for $i=1,2$. Then, by the above lemma, we have

Corollary 2. Assume that $\Pi_{0}=\varnothing$. Let $C_{1}, C_{2}$ be Weyl chambers, and $\lambda_{1} \in C_{1}, \lambda_{2} \in C_{2}$. If $\varepsilon\left(C_{1}\right)=C_{2}$, then the signatures of $\eta\left(\lambda_{1}\right)$ and $\eta\left(\lambda_{2}\right)$ are equal.

Lemma 2. Assume that $\varepsilon\left(\Pi_{0}\right)=\Pi_{0}$, and $\varepsilon\left(C_{1}\right)=C_{2}$. Then, $f_{*}\left(\mathfrak{a}_{1}^{\mathbb{C}}\right)=\mathfrak{a}_{2}^{\mathbb{C}}$, and $f_{*}\left(\mathfrak{b}_{1}^{\mathbb{C}}\right)=\mathfrak{b}_{2}^{\mathbb{C}}$.

Note that $f_{*}: \mathfrak{n}^{\mathbb{C}} \rightarrow \mathfrak{n}^{\mathbb{C}}$ induces $f_{*}: \mathbb{R}\left(\mathfrak{n}^{\mathbb{C}}\right) \rightarrow \mathbb{R}\left(\mathfrak{n}^{\mathbb{C}}\right)$. Let $\tilde{J}_{C_{i}}$ be a complex structure on $\mathbb{R}_{\mathbb{R}}\left(\mathfrak{n}^{\mathbb{C}}\right)$ corresponding to a decomposition $\mathfrak{n}^{\mathbb{C}}=\mathfrak{a}_{i}^{\mathbb{C}}+\mathfrak{b}_{i}^{\mathbb{C}}$ for each $i=1,2$. Then, we have the following:

Proposition 5. Assume that $\varepsilon\left(\Pi_{0}\right)=\Pi_{0}$, and $\varepsilon\left(C_{1}\right)=C_{2}$. Then, $f_{*}:\left(\mathbb{R}_{\mathbb{R}}\left(\mathfrak{n}^{\mathbb{C}}\right), \tilde{J}_{C_{1}}\right) \rightarrow\left(\mathbb{R}_{\mathbb{R}}\left(\mathfrak{n}^{\mathbb{C}}\right), \tilde{\boldsymbol{J}}_{C_{2}}\right)$ satisfies $f_{*} \circ \tilde{J}_{C_{1}}=\tilde{J}_{C_{2}} \circ f_{*}$.

Proof. Since $\tilde{J}_{C_{i}}=-J$ on $\mathbb{R}_{(}\left(\mathfrak{a}_{i}^{\mathbb{C}}\right)$, and $\tilde{J}_{C_{i}}=J$ on $\mathbb{R}_{(}\left(\mathfrak{b}_{i}^{\mathbb{C}}\right)$ for $i=1,2$, we have our proposition by Lemma 2 .

Now, we consider the case of a root system $A_{l}$. Let $\Pi=\left\{\alpha_{1}, \ldots, \alpha_{l}\right\}$ be a basis of $R=A_{l}$ with natural manner. Let us consider a map $f_{*}: \mathfrak{n}_{0} \rightarrow \mathfrak{n}_{0}$ defined by

$$
E_{\alpha_{i}+\cdots+\alpha_{j}} \mapsto(-1)^{j-i} E_{\varepsilon\left(\alpha_{i}+\cdots+\alpha_{j}\right)}
$$

for each $1 \leq i<j \leq l$, where $\varepsilon \in A(R)$ satisfies $\varepsilon\left(\alpha_{i}\right)=\alpha_{l-i+1}$ for $i=1, \ldots, l$.

LEMMA 3. The map $f_{*}$ is an isomorphism of $\mathrm{n}_{0}$. 
Proof. It is obvious that $f_{*}$ is bijective by definition. Recall that

$$
N_{\alpha_{i}+\cdots+\alpha_{j}, \alpha_{k}+\cdots+\alpha_{h}}= \begin{cases}1 & k=j+1 \\ 0 & \text { otherwise },\end{cases}
$$

where $i \leq j, k \leq h$, and $i \leq k$. Thus, we consider indices of top terms and last terms of roots for investigating $N_{\varepsilon\left(\alpha_{i}+\cdots+\alpha_{j}\right), \varepsilon\left(\alpha_{j+1}+\cdots+\alpha_{k}\right)}$. Note that $\varepsilon\left(\alpha_{i}\right)=\alpha_{l-i+1}$. Then, for $i \leq j, j+1 \leq k \leq l$, we have

$$
l-k+1 \leq l-(j+1)+1=l-j<l-j+1 \leq l-i+1 .
$$

Thus, we have

$$
N_{\varepsilon\left(\alpha_{i}+\cdots+\alpha_{j}\right), \varepsilon\left(\alpha_{j+1}+\cdots+\alpha_{k}\right)}=-N_{\alpha_{i}+\cdots+\alpha_{j}, \alpha_{j+1}+\cdots+\alpha_{k}}
$$

Moreover,

$$
\begin{aligned}
& {\left[(-1)^{j-i} E_{\varepsilon\left(\alpha_{i}+\cdots+\alpha_{j}\right)},(-1)^{k-j-1} E_{\varepsilon\left(\alpha_{j+1}+\cdots+\alpha_{k}\right)}\right]} \\
& \quad=(-1)^{k-i-1} N_{\varepsilon\left(\alpha_{i}+\cdots+\alpha_{j}\right), \varepsilon\left(\alpha_{j+1}+\cdots+\alpha_{k}\right)} E_{\varepsilon\left(\alpha_{i}+\cdots+\alpha_{k}\right)} \\
& \quad=(-1)^{k-i-1}\left(-N_{\alpha_{i}+\cdots+\alpha_{j}, \alpha_{j+1}+\cdots+\alpha_{k}}\right) E_{\varepsilon\left(\alpha_{i}+\cdots+\alpha_{k}\right)} \\
& \quad=(-1)^{k-i} N_{\alpha_{i}+\cdots+\alpha_{j}, \alpha_{j+1}+\cdots+\alpha_{k}} E_{\varepsilon\left(\alpha_{i}+\cdots+\alpha_{k}\right) .}
\end{aligned}
$$

The other cases are trivial. Thus, $f_{*}$ is an isomorphism.

ExAmple 1. Let $l=2$, i.e., $\Pi=\left\{\alpha_{1}, \alpha_{2}\right\}$, and $\Pi_{0}=\varnothing$. Let $C_{1}=$ $\left\{-\alpha_{1}>0, \alpha_{1}+\alpha_{2}>0\right\}$, and $C_{2}=\left\{-\alpha_{2}>0, \alpha_{1}+\alpha_{2}>0\right\}$. Then, we see

$$
f_{*}\left(E_{\alpha_{1}}\right)=E_{\alpha_{2}}, \quad f_{*}\left(E_{\alpha_{2}}\right)=E_{\alpha_{1}}, \quad f_{*}\left(E_{\alpha_{1}+\alpha_{2}}\right)=-E_{\alpha_{1}+\alpha_{2}} .
$$

Then, nilpotent Lie groups with complex structures $\tilde{\boldsymbol{J}}_{C_{1}}$ and $\tilde{\boldsymbol{J}}_{C_{2}}$ corresponding to $C_{1}$ and $C_{2}$ are

$$
N_{1}=\left\{\left(\begin{array}{ccc}
1 & \bar{z}_{1} & z_{3} \\
0 & 1 & z_{2} \\
0 & 0 & 1
\end{array}\right) \mid z_{i} \in \mathbb{C}\right\}, \quad N_{2}=\left\{\left(\begin{array}{ccc}
1 & w_{1} & w_{3}+w_{1} \bar{w}_{2} \\
0 & 1 & \bar{w}_{2} \\
0 & 0 & 1
\end{array}\right) \mid w_{i} \in \mathbb{C}\right\},
$$

respectively. We have that a holomorphic and homomorphic map $f: N_{1} \rightarrow N_{2}$ is given by

$$
w_{1}\left(z_{1}, z_{2}, z_{3}\right)=z_{2}, \quad w_{2}\left(z_{1}, z_{2}, z_{3}\right)=z_{1}, \quad w_{3}\left(z_{1}, z_{2}, z_{3}\right)=-z_{3} .
$$

Indeed, 


$$
\begin{aligned}
\left(\begin{array}{ccc}
1 & \bar{z}_{1} & z_{3} \\
0 & 1 & z_{2} \\
0 & 0 & 1
\end{array}\right) & =\exp \left(z_{2} E_{\alpha_{2}}+z_{3} E_{\alpha_{1}+\alpha_{2}}\right) \exp \left(\bar{z}_{1} E_{\alpha_{1}}\right) \\
& \stackrel{f}{\rightarrow} \exp \left(z_{2} E_{\alpha_{1}}-z_{3} E_{\alpha_{1}+\alpha_{2}}\right) \exp \left(\bar{z}_{1} E_{\alpha_{2}}\right)=\left(\begin{array}{ccc}
1 & z_{2} & -z_{3}+\bar{z}_{1} z_{2} \\
0 & 1 & \bar{z}_{1} \\
0 & 0 & 1
\end{array}\right) .
\end{aligned}
$$

REMARK 1. Except for $A_{l}, D_{l}$ and $E_{6}$, if $\sigma \in \mathfrak{a}\left(\mathfrak{g}^{\mathbb{C}}\right)$ induces a homomorphism $\sigma: \mathrm{n}^{\mathbb{C}} \rightarrow \mathrm{n}^{\mathbb{C}}$, then $\sigma\left(\mathfrak{g}_{\alpha}^{\mathbb{C}}\right)=\mathfrak{g}_{\alpha}^{\mathbb{C}}$ for each $\alpha \in R$ because $\hat{\sigma}\left(R^{+}\right)=R^{+}$, where $\hat{\sigma}(\alpha)(H)=\alpha\left(\sigma^{-1}(H)\right)$. Conversely, except for $A_{l}, D_{l}$ and $E_{6}$, if $\varphi \in$ $\left\{\varphi \in \operatorname{Aut}\left(\mathfrak{h}^{*}\right) \mid \varphi(R)=R\right\}$ satisfies $\varphi\left(R^{+}\right)=R^{+}$, then $\sigma \in \mathfrak{a}\left(\mathfrak{g}^{\mathbb{C}}\right)$ such that $\hat{\sigma}=\varphi$ satisfies $\sigma\left(\mathfrak{g}_{\alpha}^{\mathbb{C}}\right)=\mathfrak{g}_{\alpha}^{\mathbb{C}}$ for each $\alpha \in R$.

\section{Construction of nilpotent Lie algebras with a decomposition}

In this section, we construct nilpotent Lie algebras $\mathfrak{n}$ with a decomposition $\mathfrak{n}=\mathfrak{a}+\mathfrak{b}$ by root systems, and see a relation between Weyl chambers and decompositions.

Let $\Pi=\left\{\alpha_{1}, \ldots, \alpha_{l}\right\}$ be a basis of root system $R$. Let $\Pi_{0}, \Pi_{1}$ be subsets of $\Pi$. Put $\mathfrak{n}=\sum_{\alpha \in R_{\mathrm{m}}^{+}} \mathfrak{g}_{\alpha}$. Then we have the following results:

Proposition 6. Let $Q$ be a subset of $R^{+}$such that $Q$ and $R^{+} \backslash Q$ are closed. Put

$$
\mathfrak{a}=\sum_{\alpha \in R_{\mathrm{m}}^{+} \cap Q} \mathfrak{g}_{\alpha}, \quad \mathfrak{b}=\sum_{\alpha \in R_{\mathrm{m}}^{+}-Q} \mathfrak{g}_{\alpha} .
$$

Then, $\mathfrak{a}$ and $\mathfrak{b}$ are subalgebras of $\mathfrak{n}$ which satisfy $\mathfrak{n}=\mathfrak{a}+\mathfrak{b}$ and $\mathfrak{a} \cap \mathfrak{b}=\{\boldsymbol{0}\}$. Moreover, there exists a Weyl chamber $C^{\prime}$ such that $\mathfrak{a}=\sum_{\alpha \in R_{A}} \mathfrak{g}_{\alpha}, \mathfrak{b}=$ $\sum_{\alpha \in R_{B}} \mathfrak{g}_{\alpha}$, where $R_{A}=R^{+}(C) \cap R^{-}\left(C^{\prime}\right), R_{B}=R^{+}(C) \cap R^{+}\left(C^{\prime}\right)$. Conversely, let $C^{\prime}$ be a Weyl chamber. Then, $Q=R^{+} \cap R^{-}\left(C^{\prime}\right)$ is a subset of $R^{+}$such that $Q$ and $R^{+} \backslash Q$ are closed.

Proof. Because $Q$ and $R^{+} \backslash Q$ are closed, $\mathfrak{a}$ and $\mathfrak{b}$ are subalgebras of $\mathrm{n}$ which satisfy $\mathfrak{n}=\mathfrak{a}+\mathfrak{b}$ and $\mathfrak{a} \cap \mathfrak{b}=\{\boldsymbol{0}\}$. Put $Q_{1}=Q$ and $Q_{2}=R^{+} \backslash Q$. Moreover, put $R_{+}=Q_{1} \cup\left(-Q_{2}\right)$ and $R_{-}=Q_{2} \cup\left(-Q_{1}\right)$. Since $R=R_{+} \cup R_{-}$ and $R_{+} \cap R_{-}=\varnothing$, we only have to prove that $R_{+}$is closed to prove an existence of a corresponding Weyl chamber ([4]; Chapter 6, Corollary 1 of Proposition 20).

Suppose that $\alpha, \beta \in R_{+}$, and $\alpha+\beta \in R$. If $\alpha, \beta \in Q_{1}$, or $\alpha, \beta \in Q_{2}$, because $Q_{1}, Q_{2}$ are closed, then $\alpha+\beta \in Q_{1}$, or $\alpha+\beta \in Q_{2}$. Thus, suppose that $\alpha \in Q_{1}$, $\beta \in-Q_{2}$. Since $R=R_{+} \cup R_{-}$, we see that $\alpha+\beta \in R_{+}$or $\alpha+\beta \in R_{-}$. We 
shall prove $\alpha+\beta \notin R_{-}$by contradiction. Suppose that $\alpha+\beta \in R_{-}$. Then, since $R_{-}=Q_{2} \cup\left(-Q_{1}\right)$, we see that $\alpha+\beta \in Q_{2}$ or $\alpha+\beta \in-Q_{1}$. If $\alpha+\beta \in$ $-Q_{1}$, then

$$
-Q_{1} \ni-\alpha+(\alpha+\beta)=\beta \in-Q_{2},
$$

which is contrary to $\left(-Q_{1}\right) \cap\left(-Q_{2}\right)=\varnothing$. So, $\alpha+\beta \in Q_{2}$. However, then,

$$
-Q_{1} \ni-\alpha=-(\alpha+\beta)+\beta \in-Q_{2},
$$

which is contrary to $\left(-Q_{1}\right) \cap\left(-Q_{2}\right)=\varnothing$. Hence, we have $\alpha+\beta \notin R_{-}$.

Similarly, we have the following:

Proposition 7. Let $Q_{T}$ be a subset of $R_{T}^{+} \subset R_{T}=\kappa(R)$ such that $Q_{T}$ and $R_{T}^{+} \backslash Q_{T}$ are closed. Put

$$
\mathfrak{a}=\sum_{\alpha \in R_{\mathfrak{m}}^{+}, \kappa(\alpha) \in Q_{T}} \mathfrak{g}_{\alpha}, \quad \mathfrak{b}=\sum_{\alpha \in R_{\mathrm{m}}^{+}, \kappa(\alpha) \in R_{T}^{+} \backslash Q_{T}} \mathfrak{g}_{\alpha} .
$$

Then, $\mathfrak{a}$ and $\mathfrak{b}$ are subalgebras of $\mathfrak{n}$ which satisfy $\mathfrak{n}=\mathfrak{a}+\mathfrak{b}$ and $\mathfrak{a} \cap \mathfrak{b}=\{\mathbf{0}\}$.

Let

$$
Q\left(\alpha_{t}\right)=\left\{\sum_{k=1}^{t} a_{k} \alpha_{k} \in R^{+} \mid a_{t} \neq 0\right\}
$$

and

$$
Q\left(t_{1}, \ldots, t_{k}\right)=\bigcup_{t \in\left\{t_{1}, \ldots, t_{k}\right\}} Q\left(\alpha_{t}\right)
$$

for $1 \leq t_{1}<\cdots<t_{k} \leq l$.

CoROllary 3. Let $Q=Q\left(t_{1}, \ldots, t_{k}\right)=\bigcup_{t \in\left\{t_{1}, \ldots, t_{k}\right\}} Q\left(\alpha_{t}\right)$ for $1 \leq t_{1}<\cdots<$ $t_{k} \leq l$. Then, $\mathfrak{a}=\sum_{\alpha \in R_{\mathfrak{m}}^{+} \cap Q} \mathfrak{g}_{\alpha}$ and $\mathfrak{b}=\sum_{\alpha \in R_{\mathrm{m}}^{+}-Q} \mathfrak{g}_{\alpha}$ are subalgebras of $\mathrm{n}$ which satisfy $\mathfrak{n}=\mathfrak{a}+\mathfrak{b}$ and $\mathfrak{a} \cap \mathfrak{b}=\{\mathbf{0}\}$.

Proof. For $\xi=\sum_{k=1}^{t} a_{k} \alpha_{k} \in R^{+}$such that $a_{t} \neq 0$, we say that $a_{t} \alpha_{t}$ is the last term of $\xi$. Let $\xi \in Q\left(t_{1}\right), \eta \in Q\left(t_{2}\right)$, where $t_{1} \leq t_{2}$. If $\xi+\eta \in R$, then by considering the last term of $\xi+\eta$ we have $\xi+\eta \in Q\left(t_{2}\right)$. Thus, $Q$ is closed. Similarly, we have that $R^{+} \backslash Q$ is closed.

Corollary 4 ([11]). Put

$$
\mathfrak{a}\left(\Pi_{0}, \Pi_{1}\right)=\sum_{R_{\mathfrak{m}}^{+} \cap\left[\Pi_{1}\right]} \mathfrak{g}_{\alpha}, \quad \mathfrak{b}\left(\Pi_{0}, \Pi_{1}\right)=\sum_{R_{\mathfrak{m}}^{+}-\left[\Pi_{1}\right]} \mathfrak{g}_{\alpha} .
$$

Then, $\mathfrak{a}$ is a subalgebra of $\mathrm{n}$, and $\mathfrak{b}$ is an ideal of $\mathrm{n}$. 
ExAmple 2. We consider the case where $R$ is of $A_{7}$-type, and $\Pi_{0}=\left\{\alpha_{1}, \alpha_{3}\right.$, $\left.\alpha_{5}, \alpha_{7}\right\}$. Then, $R_{T}$ is of $A_{3}$-type. Thus, $R_{T}^{+}=\left\{\sum_{h=i}^{j} \xi_{h} \mid 1 \leq i \leq j \leq 3\right\}$. Let $Q_{T}=\left\{\xi_{2}, \xi_{1}+\xi_{2}\right\} \subset R_{T}^{+}$. Then, we have the following nilpotent Lie group with a complex structure:

$$
\left\{\left(\begin{array}{cccccccc}
1 & 0 & z_{13} & z_{14} & \bar{z}_{15} & \bar{z}_{16} & z_{17} & z_{18} \\
0 & 1 & z_{23} & z_{24} & \bar{z}_{25} & \bar{z}_{26} & z_{27} & z_{28} \\
0 & 0 & 1 & 0 & \bar{z}_{35} & \bar{z}_{36} & z_{37} & z_{38} \\
0 & 0 & 0 & 1 & \bar{z}_{45} & \bar{z}_{46} & z_{47} & z_{48} \\
0 & 0 & 0 & 0 & 1 & 0 & z_{57} & z_{58} \\
0 & 0 & 0 & 0 & 0 & 1 & z_{67} & z_{68} \\
0 & 0 & 0 & 0 & 0 & 0 & 1 & 0 \\
0 & 0 & 0 & 0 & 0 & 0 & 0 & 1
\end{array}\right) \mid z_{i j} \in \mathbb{C}\right\} .
$$

ExAmPle 3. Let $\left\{\alpha_{1}, \ldots, \alpha_{l}\right\}$ be a basis of the root system $R$ of $A_{l}$-type. Then,

$$
Q\left(t_{1}, \ldots, t_{k}\right)=\left\{\sum_{h=i}^{t_{j}} \alpha_{h} \mid 1 \leq i \leq t_{j}, j=1, \ldots, k\right\},
$$

for $1 \leq t_{1}<\cdots<t_{k} \leq l$.

We now consider the case where $R$ is of $A_{3}$-type. Let $\left(N, J_{0}\right)$ be the complex nilpotent Lie group defined by

$$
\left(N, J_{0}\right)=\left\{\left(\begin{array}{cccc}
1 & z_{12} & z_{13} & z_{14} \\
0 & 1 & z_{23} & z_{24} \\
0 & 0 & 1 & z_{34} \\
0 & 0 & 0 & 1
\end{array}\right) \mid z_{i j} \in \mathbb{C}\right\}
$$

and

$$
\Gamma=\left\{\left(\begin{array}{cccc}
1 & \mu_{12} & \mu_{13} & \mu_{14} \\
0 & 1 & \mu_{23} & \mu_{24} \\
0 & 0 & 1 & \mu_{34} \\
0 & 0 & 0 & 1
\end{array}\right) \mid \mu_{i j} \in \mathbb{Z}[\sqrt{-1}]\right\}
$$

Then, $\Gamma \backslash N$ is a compact complex nilmanifold. We define inductively $C^{k}(N)$ by $C^{0}(N)=N, C^{k}(N)=\left[N, C^{k-1}(N)\right]$. Then, the complex nilmanifold $\Gamma \backslash N$ is the top of a tower of holomorphic bundles with complex torus fibers $\Gamma \backslash N \rightarrow$ $C^{2}(N) \Gamma \backslash N \rightarrow C^{1}(N) \Gamma \backslash N=T_{\mathbb{C}}^{3}$, where the fibers of $\Gamma \backslash N \rightarrow C^{2}(N) \Gamma \backslash N$ and $C^{2}(N) \Gamma \backslash N \rightarrow C^{1}(N) \Gamma \backslash N$ are complex tori $T_{\mathbb{C}}^{1}$ and $T_{\mathbb{C}}^{2}$, respectively.

We now consider $N$ as a real Lie group. Let $\mathscr{C}(\mathrm{n})$ be the set of all integrable complex structures on the Lie algebra $n$ of $N$, i.e.,

$$
\mathscr{C}(\mathfrak{n})=\left\{J: \mathfrak{n} \rightarrow \mathfrak{n} \mid J^{2}=-1,[J X, J Y]=[X, Y]+J[J X, Y]+J[X, J Y]\right\} .
$$


By the above argument, we can consider the following left-invariant complex structures on the real Lie group $N$ which lie in distinct connected components of the set $\mathscr{C}(\mathrm{n})$ :

$$
\begin{aligned}
& \left\{\left(\begin{array}{cccc}
1 & z_{12} & z_{13} & z_{14} \\
0 & 1 & z_{23} & z_{24} \\
0 & 0 & 1 & z_{34} \\
0 & 0 & 0 & 1
\end{array}\right) z_{i j} \in \mathbb{C}\right\}, \\
& \left\{\left(\begin{array}{cccc}
1 & \bar{z}_{12} & z_{13} & z_{14} \\
0 & 1 & z_{23} & z_{24} \\
0 & 0 & 1 & z_{34} \\
0 & 0 & 0 & 1
\end{array}\right)\right\},\left\{\left(\begin{array}{cccc}
1 & z_{12} & \bar{z}_{13} & z_{14} \\
0 & 1 & \bar{z}_{23} & z_{24} \\
0 & 0 & 1 & z_{34} \\
0 & 0 & 0 & 1
\end{array}\right)\right\},\left\{\left(\begin{array}{cccc}
1 & z_{12} & z_{13} & \bar{z}_{14} \\
0 & 1 & z_{23} & \bar{z}_{24} \\
0 & 0 & 1 & \bar{z}_{34} \\
0 & 0 & 0 & 1
\end{array}\right)\right\}, \\
& \left\{\left(\begin{array}{ccccc}
1 & \bar{z}_{12} & \bar{z}_{13} & z_{14} \\
0 & 1 & \bar{z}_{23} & z_{24} \\
0 & 0 & 1 & z_{34} \\
0 & 0 & 0 & 1
\end{array}\right)\right\},\left\{\left(\begin{array}{cccc}
1 & \bar{z}_{12} & z_{13} & \bar{z}_{14} \\
0 & 1 & z_{23} & \bar{z}_{24} \\
0 & 0 & 1 & \bar{z}_{34} \\
0 & 0 & 0 & 1
\end{array}\right)\right\},\left\{\left(\begin{array}{cccc}
1 & z_{12} & \bar{z}_{13} & \bar{z}_{14} \\
0 & 1 & \bar{z}_{23} & \bar{z}_{24} \\
0 & 0 & 1 & \bar{z}_{34} \\
0 & 0 & 0 & 1
\end{array}\right)\right\}, \\
& \left\{\left(\begin{array}{cccc}
1 & \bar{z}_{12} & \bar{z}_{13} & \bar{z}_{14} \\
0 & 1 & \bar{z}_{23} & \bar{z}_{24} \\
0 & 0 & 1 & \bar{z}_{34} \\
0 & 0 & 0 & 1
\end{array}\right)\right\} .
\end{aligned}
$$

Indeed, consider $T_{\mathbb{C}}^{1}$ and $T_{\mathbb{C}}^{2}$ and the base space $T_{\mathbb{C}}^{3}$ as real manifolds. Let us consider the orientations induced on real tori $T_{\mathbb{C}}^{1}, T_{\mathbb{C}}^{2}$ and $T_{\mathbb{C}}^{3}$ of the tower by the above invariant complex structures on $\Gamma \backslash N$. Note that $\Gamma C^{k}(N)=C^{k}(N) \Gamma$. Moreover, note that elements of $N$ and $C^{1}(N)$ can be written by elements of $C^{1}(N), C^{2}(N)$, and elements of special-type as follows:

$$
N \ni\left(\begin{array}{cccc}
1 & z_{12} & z_{13} & z_{14} \\
0 & 1 & z_{23} & z_{24} \\
0 & 0 & 1 & z_{34} \\
0 & 0 & 0 & 1
\end{array}\right)=\left(\begin{array}{cccc}
1 & 0 & z_{13} & z_{14}-z_{13} z_{34} \\
0 & 1 & 0 & z_{24} \\
0 & 0 & 1 & 0 \\
0 & 0 & 0 & 1
\end{array}\right)\left(\begin{array}{cccc}
1 & z_{12} & 0 & 0 \\
0 & 1 & z_{23} & 0 \\
0 & 0 & 1 & z_{34} \\
0 & 0 & 0 & 1
\end{array}\right)
$$

and

$$
C^{1}(N) \ni\left(\begin{array}{cccc}
1 & 0 & z_{13} & z_{14} \\
0 & 1 & 0 & z_{24} \\
0 & 0 & 1 & 0 \\
0 & 0 & 0 & 1
\end{array}\right)=\left(\begin{array}{cccc}
1 & 0 & 0 & z_{14} \\
0 & 1 & 0 & 0 \\
0 & 0 & 1 & 0 \\
0 & 0 & 0 & 1
\end{array}\right)\left(\begin{array}{cccc}
1 & 0 & z_{13} & 0 \\
0 & 1 & 0 & z_{24} \\
0 & 0 & 1 & 0 \\
0 & 0 & 0 & 1
\end{array}\right)
$$

Thus, we have that the above invariant complex structures lie in distinct connected components of the set $\mathscr{C}(\mathrm{n})$. 


\section{Hodge numbers}

In this section, we recall relations between the decomposition $\mathfrak{g}=\mathfrak{a}+\mathfrak{b}$ and $h^{s, t}\left(\mathfrak{g}_{\tilde{J}}\right)=\operatorname{dim} H_{\tilde{\hat{o}}}^{s, t}\left(\mathfrak{g}_{\tilde{J}}\right)$ in the previous paper [12].

Let $\mathfrak{g}$ be a real Lie algebra with a direct decomposition

$$
\mathfrak{g}=\mathfrak{a}+\mathfrak{b},
$$

where $\mathfrak{a}$ and $\mathfrak{b}$ are Lie subalgebras of $\mathfrak{g}$. Take bases of the Lie subalgebras $\mathfrak{a}$ and $\mathfrak{b}$ :

$$
\begin{aligned}
\mathfrak{a} & =\operatorname{span}_{\mathbb{R}}\left\{U_{1}, \ldots, U_{p}\right\}, \\
\mathfrak{b} & =\operatorname{span}_{\mathbb{R}}\left\{V_{1}, \ldots, V_{q}\right\} .
\end{aligned}
$$

Let $\left\{\alpha_{1}, \ldots, \alpha_{p}, \beta_{1}, \ldots, \beta_{q}\right\}$ be the dual basis of $\left\{U_{1}, \ldots, U_{p}, V_{1}, \ldots, V_{q}\right\}$. We can assume that

$$
d \alpha_{i}=-\sum_{k, h} C_{k h}^{i} \alpha_{k} \wedge \alpha_{h}-\sum_{k, s} D_{k s}^{i} \alpha_{k} \wedge \beta_{s}, \quad d \beta_{j}=-\sum_{s, t} E_{s t}^{j} \beta_{s} \wedge \beta_{t}-\sum_{k, s} F_{k s}^{j} \alpha_{k} \wedge \beta_{s}
$$

for each $i, j$, where $C_{k h}^{i}, D_{k s}^{i}, E_{s t}^{j}, F_{k s}^{j} \in \mathbb{R}$.

Let $\mathfrak{g}_{\mathfrak{a}}, \mathfrak{g}_{\mathfrak{b}}$ be real Lie algebras such that $\mathfrak{g}_{\mathfrak{a}}^{*}=\operatorname{span}\left\{\mu_{1}^{0}, \ldots, \mu_{p}^{0}, v_{1}^{0}, \ldots, v_{q}^{0}\right\}$ and $\mathfrak{g}_{\mathfrak{b}}^{*}=\operatorname{span}\left\{\mu_{1}^{1}, \ldots, \mu_{p}^{1}, v_{1}^{1}, \ldots, v_{q}^{1}\right\}$ have the structure equations

$$
\begin{array}{rlrl}
d \mu_{i}^{0} & =-\sum_{k, s} D_{k s}^{i} \mu_{k}^{0} \wedge v_{s}^{0}, & d v_{j}^{0}=-\sum_{s, t} E_{s t}^{j} v_{s}^{0} \wedge v_{t}^{0}, \\
d \mu_{i}^{1}=-\sum_{k, h} C_{k h}^{i} \mu_{k}^{1} \wedge \mu_{h}^{1}, & d v_{j}^{1}=-\sum_{k, s} F_{k s}^{j} \mu_{k}^{1} \wedge v_{s}^{1},
\end{array}
$$

respectively. Since $\bar{\partial}^{2}=0$ on $\bigwedge_{\tilde{J}}^{*, *}\left(\mathfrak{g}^{\mathbb{C}}\right)^{*}$, we have that $d^{2}=0$ on $\bigwedge^{1} \mathfrak{g}_{\mathfrak{a}}^{*}$ and $\bigwedge^{1} \mathfrak{g}_{\mathfrak{b}}^{*}$, which implies that $\mathfrak{g}_{\mathfrak{a}}, \mathfrak{g}_{\mathfrak{b}}$ are Lie algebras (cf. [12]). Then we have

THEOREM 7 ([12]). For each $r$,

$$
\sum_{s+t=r} h^{s, t}\left(\mathfrak{g}_{\tilde{J}}\right)=\operatorname{dim} H^{r}\left(\mathfrak{g}_{\mathfrak{a}} \times \mathfrak{g}_{\mathfrak{b}}\right) .
$$

\section{Symmetry of summations}

In this section, we prove a property of $\sum_{s+t=r} h^{s, t}\left(n_{\tilde{J}}\right)$ for the case of a root system $A_{l}$.

Let $\Pi=\left\{\alpha_{1}, \ldots, \alpha_{l}\right\}$ be a basis of the root system $R$ of $A_{l}$-type, and $\Pi_{0}$ a subset of $\Pi$. Let

$$
\Pi_{1, k}=\left\{\alpha_{1}, \ldots, \alpha_{k}\right\}, \quad \Pi_{2, k}=\left\{\alpha_{k}, \ldots, \alpha_{l}\right\} .
$$


Then, by the equations (1), (2), we have

$$
\begin{aligned}
\mathfrak{g}_{\mathfrak{a}\left(\Pi_{0}, \Pi_{1, k-1}\right)} & =\sum_{\alpha \in R_{\mathfrak{m}}^{+}-\left[\Pi_{1, k-1}\right]} \mathfrak{g}_{\alpha} \times \mathbb{R}^{\sharp\left(R_{\mathfrak{m}} \cap\left[\Pi_{1, k-1}\right]\right)}, \\
\mathfrak{g}_{\mathfrak{b}\left(\Pi_{0}, \Pi_{1, k-1}\right)} & =\sum_{\alpha \in R_{\mathfrak{m}}^{+}-\left[\Pi_{2, k+1}\right]} \mathfrak{g}_{\alpha} \times \mathbb{R}^{\sharp\left(R_{\mathfrak{m}} \cap\left[\Pi_{2, k+1}\right]\right)}, \\
\mathfrak{g}_{\mathfrak{a}\left(\Pi_{0}, \Pi_{2, k+1}\right)} & =\sum_{\alpha \in R_{\mathfrak{m}}^{+}-\left[\Pi_{2, k+1}\right]} \mathfrak{g}_{\alpha} \times \mathbb{R}^{\sharp\left(R_{\mathfrak{m}} \cap\left[\Pi_{2, k+1}\right]\right)}, \\
\mathfrak{g}_{\mathfrak{b}\left(\Pi_{0}, \Pi_{2, k+1}\right)} & =\sum_{\alpha \in R_{\mathfrak{m}}^{+}-\left[\Pi_{1, k-1}\right]} \mathfrak{g}_{\alpha} \times \mathbb{R}^{\sharp\left(R_{\mathfrak{m}} \cap\left[\Pi_{1, k-1}\right]\right)} .
\end{aligned}
$$

Indeed, let us consider second equations $d v_{j}^{1}=-\sum_{k, s} F_{k s}^{j} \mu_{k}^{1} \wedge v_{s}^{1}$ of (2). Let $i \leq k \leq j$. Then, we see

$$
\sum_{s=i}^{j} \alpha_{s} \in\left(R^{+} \backslash\left[\Pi_{1, k-1}\right]\right) \cap\left(R^{+} \backslash\left[\Pi_{2, k+1}\right]\right) .
$$

Consider elements

$$
\sum_{s=m}^{i-1} \alpha_{s} \in\left[\Pi_{1, k-1}\right] \cap\left(R^{+} \backslash\left[\Pi_{2, k+1}\right]\right)
$$

where $m \leq i-1$. Then, we have

$$
\sum_{s=m}^{i-1} \alpha_{s}+\sum_{s=i}^{j} \alpha_{s}=\sum_{s=m}^{j} \alpha_{s} \in\left(R^{+} \backslash\left[\Pi_{1, k-1}\right]\right) \cap\left(R^{+} \backslash\left[\Pi_{2, k+1}\right]\right) .
$$

Then, by the equation (2), we have

$$
\mathfrak{g}_{\mathfrak{b}\left(\Pi_{0}, \Pi_{1, k-1}\right)}=\sum_{\alpha \in R_{\mathfrak{m}}^{+}-\left[\Pi_{2, k+1}\right]} \mathfrak{g}_{\alpha}
$$

Similarly, we have

$$
\mathfrak{g}_{\mathfrak{b}\left(\Pi_{0}, \Pi_{2, k+1}\right)}=\sum_{\alpha \in R_{\mathrm{m}}^{+}-\left[\Pi_{1, k-1}\right]} \mathfrak{g}_{\alpha} \times \mathbb{R}^{\sharp\left(R_{\mathfrak{m}} \cap\left[\Pi_{1, k-1}\right]\right)} .
$$

Thus, we have

$$
\mathfrak{g}_{\mathfrak{a}\left(\Pi_{0}, \Pi_{1, k-1}\right)}=\mathfrak{g}_{\mathfrak{b}\left(\Pi_{0}, \Pi_{2, k+1}\right)}, \mathfrak{g}_{\mathfrak{b}\left(\Pi_{0}, \Pi_{1, k-1}\right)}=\mathfrak{g}_{\mathfrak{a}\left(\Pi_{0}, \Pi_{2, k+1}\right)} .
$$


Hence, we have

$$
\mathfrak{g}_{\mathfrak{a}\left(\Pi_{0}, \Pi_{1, k-1}\right)} \times \mathfrak{g}_{\mathfrak{b}\left(\Pi_{0}, \Pi_{1, k-1}\right)} \cong \mathfrak{g}_{\mathfrak{a}\left(\Pi_{0}, \Pi_{2, k+1}\right)} \times \mathfrak{g}_{\mathfrak{b}\left(\Pi_{0}, \Pi_{2, k+1}\right)} .
$$

Thus, we have

THEOREM 8. Let $\tilde{J}_{1, k}, \tilde{J}_{2, k}$ be complex structures on $\mathbb{R}_{\mathbb{R}}\left(\mathfrak{n}^{\mathbb{C}}\right)$ corresponding to decompositions induced by $\Pi_{1, k}, \Pi_{2, k}$, respectively. Then,

$$
\sum_{s+t=r} h^{s, t}\left(\mathfrak{n}_{\tilde{J}_{1, k-1}}\right)=\sum_{s+t=r} h^{s, t}\left(\mathfrak{n}_{\tilde{J}_{2, k+1}}\right)
$$

for each $k$ and $r$.

As an application, we have the following:

Proposition 8 ([11]). Let $\Pi_{0}$ be a subset of $\Pi$ such that $\varepsilon\left(\Pi_{0}\right)=\Pi_{0}$. Let $\tilde{\boldsymbol{J}}_{k}$ be complex structures on $\mathbb{R}_{\mathbb{R}}\left(\mathrm{n}^{\mathbb{C}}\right)$ corresponding to decompositions induced by $\Pi_{1, k}$ for each $k=0, \ldots, l$. Then,

$$
\sum_{s+t=r} h^{s, t}\left(\mathfrak{n}_{\tilde{J}_{k}}\right)=\sum_{s+t=r} h^{s, t}\left(\mathfrak{n}_{\tilde{J}_{l-k-1}}\right)
$$

for each $k$ and $r$.

Proof. Since $\varepsilon\left(\Pi_{0}\right)=\Pi_{0}$, we have

$$
\begin{aligned}
\sum_{s+t=r} h^{s, t}\left(\mathfrak{n}_{\tilde{J}_{k}}\right) & =\sum_{s+t=r} h^{s, t}\left(\mathrm{n}_{\tilde{J}_{1, k}}\right)=\sum_{s+t=r} h^{s, t}\left(\mathfrak{n}_{\tilde{J}_{2, k+2}}\right) \\
& =\sum_{s+t=r} h^{s, t}\left(\mathfrak{n}_{\tilde{J}_{1, l-k-1}}\right)=\sum_{s+t=r} h^{s, t}\left(\mathfrak{n}_{\tilde{J}_{l-k-1}}\right)
\end{aligned}
$$

by considering a holomorphic isomorphism $f_{*}:\left(\mathbb{R}_{\mathbb{R}}\left(\mathrm{n}^{\mathbb{C}}\right), \tilde{J}_{2, k+2}\right) \rightarrow\left(\mathbb{R}_{\mathbb{R}}\left(\mathrm{n}^{\mathbb{C}}\right)\right.$, $\left.\tilde{J}_{1, l-k-1}\right)$ (see Proposition 5; note that $\varepsilon\left(\alpha_{k+2}\right)=\alpha_{l-k-1}$ and $\left.\varepsilon\left(\alpha_{l}\right)=\alpha_{1}\right)$.

ExAmple 4. Let $\Pi=\left\{\alpha_{1}, \ldots, \alpha_{6}\right\}$, and $\Pi_{0}=\left\{\alpha_{4}\right\}$. Then, $\mathbb{R}_{\mathbb{R}}\left(\mathfrak{n}^{\mathbb{C}}\right)=$ $\operatorname{span}\left\{E_{\alpha}, F_{\alpha}\right\}_{\alpha \in R_{m}^{+}}$is a real 40-dimensional nilpotent Lie algebra with the structure equations $\left[E_{\alpha}, E_{\beta}\right]=E_{\alpha+\beta},\left[E_{\alpha}, F_{\beta}\right]=\left[F_{\alpha}, E_{\beta}\right]=F_{\alpha+\beta},\left[F_{\alpha}, F_{\beta}\right]=-E_{\alpha+\beta}$ for $\alpha, \beta, \alpha+\beta \in R_{\mathfrak{m}}^{+}$, where $\alpha=\sum_{h=i_{\alpha}}^{j_{\alpha}} \alpha_{h}$ and $\beta=\sum_{h=i_{\beta}}^{j_{\beta}} \alpha_{h}$ with $i_{\alpha}<i_{\beta}$. Then, in the case of $k=4, \tilde{J}_{1,3}$ satisfies $\tilde{J}_{1,3} E_{\alpha}=-F_{\alpha}\left(\tilde{J}_{1,3} F_{\alpha}=E_{\alpha}\right)$ for $\alpha \in\left\{\alpha_{1}, \alpha_{2}, \alpha_{3}\right.$, $\left.\alpha_{1}+\alpha_{2}, \alpha_{2}+\alpha_{3}, \alpha_{1}+\alpha_{2}+\alpha_{3}\right\}$ and otherwise $\tilde{J}_{1,3} E_{\alpha}=F_{\alpha} \quad\left(\tilde{J}_{1,3} F_{\alpha}=-E_{\alpha}\right)$. On the other hand, $\tilde{J}_{2,5}$ satisfies $\tilde{J}_{2,5} E_{\alpha}=-F_{\alpha}$ for $\alpha \in\left\{\alpha_{5}, \alpha_{6}, \alpha_{5}+\alpha_{6}\right\}$ and otherwise $\tilde{J}_{2,5} E_{\alpha}=F_{\alpha}$. Then,

$$
\sum_{s+t=r} h^{s, t}\left(\mathfrak{n}_{\tilde{J}_{1,3}}\right)=\sum_{s+t=r} h^{s, t}\left(\mathfrak{n}_{\tilde{J}_{2,5}}\right) .
$$


REMARK 2. In general, $\mathfrak{g}_{\mathfrak{a}}$ and $\mathfrak{g}_{\mathfrak{b}}$ have different type. For example, let us consider the case of $B_{3}$. Let $\left\{\alpha_{1}, \alpha_{2}, \alpha_{3}\right\}$ be a basis of the root system $R$ of $B_{3}$-type with natural manner. Let $\Pi_{0}=\varnothing$, and $\Pi_{1}=\left\{\alpha_{1}\right\}$. Then, we have

$$
\mathfrak{g}_{\mathfrak{a}}=\sum_{\alpha \in R^{+}-\left[\Pi_{1}\right]} \mathfrak{g}_{\alpha} \times \mathbb{R}^{1}, \quad \mathfrak{g}_{\mathfrak{b}}=\sum_{\alpha \in R_{A_{3}}^{+}-\left\{\alpha_{3}\right\}} \mathfrak{g}_{\alpha} \times \mathbb{R}^{4},
$$

where $R_{A_{3}}^{+}=\left\{\alpha_{1}, \alpha_{2}, \alpha_{3}, \alpha_{1}+\alpha_{2}, \alpha_{2}+\alpha_{3}, \alpha_{1}+\alpha_{2}+\alpha_{3}\right\}$, and we define $\left[E_{\alpha}, E_{\beta}\right]=0$ for $\alpha, \beta \in R_{A_{3}}^{+}$such that $\alpha+\beta \notin R_{A_{3}}^{+}$.

Example 5. Let $\Pi_{0}=\left\{\alpha_{2}, \ldots, \alpha_{l-1}\right\}$. Then, $\varepsilon\left(\Pi_{0}\right)=\Pi_{0}$, and $\mathrm{n}^{\mathbb{C}}$ is a complex (2l-1)-dimensional Heisenberg algebra. Then, $\sum_{s+t=r} h^{s, t}\left(\mathfrak{n}_{\tilde{J}_{k}}\right)=$ $\sum_{s+t=r} h^{s, t}\left(\mathfrak{n}_{\tilde{J}_{l-k-1}}\right)$ for each $k$ and $r$.

\section{Acknowledgement}

The author would like to express his deep appreciation to Professor Yusuke Sakane for valuable advice and encouragement during his preparation of the paper.

\section{References}

[1] D. V. Alekseevsky, Flag manifolds, 11th Yugoslav Geometrical Seminar (Divčibare, 1996), Zb. Rad. Mat. Inst. Beograd. (N. S.), 6 (14) (1997), 3-35.

[2] A. Andrada and S. Salamon, Complex product structures on Lie algebras, Forum Math. 17 (2005), 261-295.

[3] A. I. Arvanitoyeorgos, I. Chrysikos and Y. Sakane, Homogeneous Einstein metrics on generalized flag manifolds $S p(n) /(U(p) \times U(q) \times S p(n-p-q))$, Proceedings of the 2nd International Colloquium on Differential Geometry and its Related Fields, 1-24, World Sci. Publ., Hackensack, NJ, 2012.

[4] N. Bourbaki, Groupes et algèbres de Lie, Élements de Mathématique, Masson Publishing, Paris, 1981.

[5] S. Console and A. Fino, Dolbeault cohomology of compact nilmanifolds, Transform. Groups 6 (2001), 111-124.

[6] M. S. Raghunathan, Discrete subgroups of Lie groups, Ergebnisse der Mathematik und ihrer Grenzgebiete, Band 68, Springer-Verlag, New York-Heidelberg, ix +227 pp. (1972).

[7] Y. Sakane, On compact complex parallelisable solvmanifolds, Osaka J. Math. 13 (1976), $187-212$.

[8 ] S. M. Salamon, Complex structures on nilpotent Lie algebras, J. Pure. Appl. Algebra 157 (2001), 311-333.

[9] T. Yamada, Invariant pseudo-Kähler metrics on generalized flag manifolds, Differential Geom. Appl. 36 (2014), 44-55.

[10] T. Yamada, Remarks on Hodge numbers and invariant complex structures of compact nilmanifolds, Complex manifolds 3 (2016), 271-281. 
[11] T. Yamada, Some relations between Hodge numbers and invariant complex structures on compact nilmanifolds, Complex manifolds 4 (2017), 73-83.

[12] T. Yamada, Complex structures on the complexification of a real Lie algebra, Complex manifolds 5 (2018), 150-157.

\author{
Takumi Yamada \\ Department of Mathematics \\ Interdisciplinary Faculty of Science and Engineering \\ Shimane University \\ Nishikawatsu-cho, Matsue City, Shimane, 690-8504, Japan \\ E-mail: t_yamada@riko.shimane-u.ac.jp
}

